takes place only once the tubes are in the laboratory. If we consider only the samples sent from regional or remote sources, the rate of indeterminate test result is still very low (one $(0.3 \%)$ out of 317$)$. The use of T-Cell Xtend ${ }^{\mathrm{TM}}$ for all samples shipped overnight is likely to have had a beneficial effect on older samples. The cold weather conditions had no influence on the rate of indeterminate results (all samples were sent and processed in winter). The number of tests performed in very young or very old patients being small, we cannot assess the rate of indeterminate in these population groups.

The slightly higher rate of positivity in samples referred from Vaud county (17\% versus $10 \%$ in other groups) can be explained since these samples were mostly from recent immigrants from countries with a high prevalence of tuberculosis, whereas the samples from the city or from remote places were more often taken for contact tracing investigations where the rate of infection may be expected to be lower.

In conclusion, these results demonstrate that the use of lithium heparinate blood collection tubes for all samples and T-Cell $X_{t e n d^{\mathrm{TM}}}$ for samples shipped overnight allows the processing of samples from all over Switzerland while maintaining the rate of indeterminate test results at a very low level in spite of longer travel time (8-36 h). In our experience, the only indeterminate test results are observed in patients with some immune abnormality and do not represent a laboratory failure.

\section{A. Zellweger* and J-P. Zellweger ${ }^{\#}$}

*Meditest Laboratory, Lausanne, "Chest physician, Fribourg, Switzerland.

Correspondence: J-P. Zellweger, Chest physician, Rue de Locarno 1, 1700 Fribourg, Switzerland. E-mail: zellwegerjp@ swissonline.ch

Statement of Interest: A statement of interest for J-P. Zellweger can be found at www.erj.ersjournals.com/site/misc/ statements.xhtml

\section{REFERENCES}

1 Beffa $\mathrm{P}$, Zellweger A, Janssens J-P, et al. Indeterminate test results of T-SPOT ${ }^{\mathrm{TM}}$.TB performed under routine field conditions. Eur Respir J 2008; 31: 842-846.

2 Wang SH, Powell DA, Nagaraja HN, et al. Evaluation of a modified interferon- $\gamma$ release assay for the diagnosis of latent tuberculosis infection in adult and paediatric populations that enables delayed processing. Scand J Infect Dis 2010; 42: 845-850.

3 Oxford Immunotec. T-SPOT®.TB. www.oxfordimmunotec.com/ T-SPOT_International Date last accessed: March 10, 2011. Date last updated: 2010.

\title{
Is MDR-TB on the rise in Mozambique? Results of a national drug resistance survey
}

\section{To the Editors:}

Tuberculosis (TB) remains a serious public health problem in many low- and middle-income countries in Africa, Asia and the former Soviet Union [1]. Mozambique is one of them. Despite the fact that in this country detection of patients with TB has drastically increased during the past decade due to improved notification (from nearly 20,000 new TB cases notified in 1998 to nearly 40,000 in 2008), less than half of the cases of TB estimated to emerge annually are currently being detected. As in other southern African countries, the HIV epidemic is fuelling the TB epidemic in Mozambique, with $60 \%$ of patients with TB being co-infected with HIV [1]. Drug-resistant TB is thought to be a major problem, although the latest information available on the magnitude of resistance to anti-TB drugs is from 1998, when the first national drug resistance survey (DRS) was conducted. At that time, multidrug-resistant (MDR)-TB (defined as TB resistant to at least isoniazid and rifampicin, the two main first-line drugs in the treatment of TB) was found among 3.5 and 3.3\% of new and previously treated TB cases, respectively [2]. Since then, there has been no further investigation of the magnitude of drug resistance in the country. Based on the latest available data, $>3,500$ MDR-TB cases are estimated to have emerged in Mozambique in 2008 [3]. Detecting and treating patients with MDR-TB is feasible but substantially more complex and costly than treating patients with fully susceptible Mycobacterium tuberculosis strains. Knowing the levels of anti-TB drug resistance in the country is essential for any national TB programme (NTP) to evaluate the efficacy of TB control measures and treatment practices in the community, as well as to design effective treatment regimens for all patients with TB $[4,5]$.

For this reason, after nearly 10 yrs from the first survey, a new DRS on a national representative sample of TB patients has been conducted, with the objectives to investigate levels and patterns of resistance to first- and second-line anti-TB drugs among new and previously treated TB cases, compare the new findings with the existing data, and explore the association between the HIV epidemic and anti-TB drug resistance. The findings will be used by the NTP to develop treatment and management policies for TB and MDR-TB patients.

The survey was designed according to the guidelines of the World Health Organization (WHO) [6], and conducted between February 2007 and May 2008 in 40 diagnostic centres sampled from all centres distributed in all 11 provinces of the country. The sampling was probability proportional to size, based on the number of new sputum smear-positive cases in 2006. Each cluster was required to enrol 30 new sputum smear-positive TB patients. During the enrolment period, all previously treated TB patients presenting to the 40 diagnostic 
centres were also included. Sputum smear-negative and extrapulmonary TB patients were excluded from the survey. Patients were classified as newly diagnosed or previously treated according to $\mathrm{WHO}$ definitions [6]. The following variables were collected through a questionnaire administered during sputum collection: sex, age, treatment history (new/ previously treated) and HIV status. Medical records were reviewed to confirm the reliability of the information gathered through the questionnaire. Sputum samples were sent to the National Reference Laboratory in Maputo, Mozambique, for culture and first-line drug susceptibility testing (DST), performed on Löwenstein-Jensen medium. All MDR-TB strains and $10 \%$ of randomly selected susceptible strains were sent to the Supranational Reference Laboratory in Milan, Italy for re-checking and second-line DST. The study was approved by the National Ethical Review Board of Ministry of Health of Mozambique. Informed consent was obtained before enrolment. Summary analyses were performed using Stata (version 10.0; StataCorp, College Station, TX, USA).

A total of 1,398 sputum smear-positive pulmonary TB patients were diagnosed during the study period. Due to failure of TB culture growth, 198 of them were excluded from the analysis (159 were culture-negative and 39 were culture-contaminated). Of the 1,200 cases with culture-positive specimens, DST results were available for 1,127 patients (in 21 cases DST was contaminated and in 52 cases the secondary culture did not grow). The overall total culture and DST recovery rate was $80.6 \%$, which was consistent with national expectations and $\mathrm{WHO}$ recommendations [6].

Of the 1,127 patients with final results, the vast majority were new cases of TB, as only 25 previously treated cases could be included in the survey $(2.2 \%$ of the total, consistent with national notification data). As expected, males were more frequent than females $(61.8 \%)$. The mean \pm SD age of the study population was $35 \pm 13$ yrs. Information on HIV status was available for 937 patients $(83.1 \%$ of those with final DST results), and $36.9 \%$ of them (346 out of 937 cases) had HIV coinfection.

Overall proportions of MDR-TB were 3.5\% (38 out of 1,102, $95 \%$ CI $2.2-4.8 \%$ ) among newly diagnosed TB cases and $11.2 \%$ (three out of $25,95 \%$ CI $0.0-25.2 \%$ ) among those previously

TABLE 1 Prevalence of resistance to first-line antituberculosis drugs among patients with sputum smear-positive pulmonary tuberculosis (TB)

\begin{tabular}{|c|c|c|c|c|}
\hline Drug-resistance pattern $\mathrm{n}$ & 1102 & 25 & & \\
\hline Resistance to any drug & $122(11.3,9.3-13.2)$ & $6(23.3,6.6-40.2)$ & $2.2(1.1-4.4)$ & 0.044 \\
\hline \multicolumn{5}{|l|}{ Any resistance to } \\
\hline $\mathrm{H}$ & $85(7.8,6.0-9.6)$ & $4(15.0,0.0-31.0)$ & $2.1(0.8-5.2)$ & 0.129 \\
\hline S & $80(7.4,5.9-9.0)$ & $3(9.8,0.0-22.1)$ & $1.6(0.6-4.9)$ & 0.370 \\
\hline \multicolumn{5}{|l|}{ Monoresistance to } \\
\hline $\mathrm{H}$ & $31(2.8,1.8-3.8)$ & $0(0)$ & $0(0)$ & 0.395 \\
\hline $\mathrm{R}$ & $2(0.2,0.0-0.5)$ & $2(8.4,0.0-19.5)$ & $44.1(6.5-300.5)$ & 0.000 \\
\hline E & $0(0)$ & $0(0)$ & & \\
\hline HRE & $1(0.1,0.0-0.3)$ & $0(0)$ & $0(0)$ & 0.880 \\
\hline HRS & $18(1.6,0.7-2.6)$ & $0(0)$ & $0(0)$ & 0.520 \\
\hline HRES & $11(1.1,0.3-2.0)$ & $2(6.0,0.0-15.4)$ & $8.0(1.9-34.3)$ & 0.001 \\
\hline Total & $38(3.5,2.2-4.8)$ & $3(11.2,0.0-25.2)$ & $3.5(1.2-10.5)$ & 0.024 \\
\hline \multicolumn{5}{|l|}{ Other patterns } \\
\hline $\mathrm{HE}$ & $0(0)$ & $0(0)$ & & \\
\hline HS & $14(1.2,0.6-1.9)$ & $1(3.8,0.0-12.1)$ & $3.1(0.4-23.0)$ & 0.239 \\
\hline ES & $1(0.1,0.0-0.3)$ & $0(0)$ & $0(0)$ & 0.880 \\
\hline HES & $2(0.2,0.0-0.5)$ & $0(0)$ & $0(0)$ & 0.831 \\
\hline RE & $0(0)$ & $0(0)$ & & \\
\hline RS & $0(0)$ & $0(0)$ & & \\
\hline
\end{tabular}

Data are presented as $n(\%, 95 \% \mathrm{Cl})$, unless otherwise stated. Risk ratio (RR) was calculated to access risk difference between patients newly diagnosed and previously treated for TB. H: isoniazid; R: rifampicin; E: ethambutol; S: streptomycin. 
treated for TB. Levels of resistance to isoniazid $(\mathrm{H})$, rifampicin (R), ethambutol (E) and streptomycin (S), as well as frequencies of any resistance, monoresistance and resistance to other patterns are shown by treatment history in table 1 . All 41 MDR-TB strains were tested for second-line drug resistance and no cases of extensively drug-resistant (XDR)-TB (defined as MDR-TB plus resistance to a fluoroquinolone and at least one second-line injectable agent (amikacin, kanamycin and/or capreomycin)) were observed.

Among the 917 new TB cases with known HIV status, proportions of MDR-TB were 2.1\% (12 out of 583, 95\% CI $0.9-3.4 \%$ ) and $4.5 \%$ (15 out of $334,95 \%$ CI $2.2-6.7 \%$ ) among those with negative and positive HIV status, respectively. MDR-TB frequencies were higher in people living with HIV, with risk ratio 2.4 (95\% CI $1.2-4.8 ; \mathrm{p}=0.007)$. The association between resistance to any first-line anti-TB drug and HIV was not found to be statistically significant (table 2).

Compared with a study conducted recently in neighbouring Tanzania, where MDR-TB was found in only $1.1 \%$ of the new TB cases tested [7], and surveys conducted in several African countries in the last decade [8], the proportion of MDRTB detected in Mozambique is quite high. Fortunately, no significant difference could be detected between the levels of MDR-TB found in new TB cases in 1998 (3.5\%, 95\% CI 2.5$4.8 \%)$ and in 2007 (3.5\%, 95\% CI 2.2-4.8\%). This very important finding confirms that MDR-TB is not rapidly spreading in the community and that the NTP has been effective in preventing the development and transmission of drug resistance by improving detection of $\mathrm{TB}$ cases and provision of proper treatment. Due to the low number of previously treated cases enrolled in the study, it is not possible to conclude whether the proportion of MDR-TB is increasing in this group (four out of 122 in 1998 versus three out of 25 in 2007).

People living with HIV were found to have a statistically significant greater risk of being infected with MDR-TB strains compared with HIV-negative patients (RR 2.4, 95\% CI 1.2-4.8; $\mathrm{p}=0.007)$. As shown by reports from the neighboring South African province of Kwazulu Natal [9, 10], patients with MDR/XDR-TB have very high case fatality if they are not

\begin{tabular}{|c|c|c|c|}
\hline \multirow[t]{2}{*}{ TABLE 2} & \multicolumn{3}{|c|}{$\begin{array}{l}\text { Statistically significant differences in rates of any } \\
\text { resistance and multidrug-resistant (MDR) } \\
\text { tuberculosis (TB) by HIV status among new cases }\end{array}$} \\
\hline & & Any resistance & MDR \\
\hline \multicolumn{2}{|c|}{$\begin{array}{l}\text { Resistant cases in HIV-negative } \\
\text { TB patients }\end{array}$} & $56 / 583(9.6)$ & 12/583 (2.1) \\
\hline \multicolumn{2}{|c|}{$\begin{array}{l}\text { Resistant cases in HIV-positive } \\
\text { TB patients }\end{array}$} & 42/334 (12.6) & $15 / 334(4.5)$ \\
\hline \multicolumn{2}{|l|}{ RR (95\% Cl) } & $1.3(0.9-1.9)$ & $2.4(1.2-4.8)$ \\
\hline \multicolumn{2}{|l|}{$p$-value } & 0.100 & 0.007 \\
\hline
\end{tabular}

rapidly diagnosed and treated with second-line anti-TB drugs and antiretroviral therapy.

In Mozambique, second-line treatment for MDR-TB is available to the entire country free of charge through the NTP, although the majority of patients accessing treatment live in Maputo. More efforts are needed to ensure rapid diagnosis of MDR-TB and access to treatment with second-line drugs, as well as antiretroviral therapy for all those in need.

\section{P. Samo Gudo*, Z. Cuna*, E. Coelho", S. Maungate", E. Borroni ${ }^{\top}$, P. Miotto ${ }^{\top}$, S. Ahmadova ${ }^{+}$, M. Brouwer ${ }^{\S}$, G.B. Migliori ${ }^{f}$, M. Zignol** and D.M. Cirillo}

*Ministry of Health, "National Reference Tuberculosis Laboratory, ${ }^{+}$World Health Organization, ${ }^{\S}$ Health Alliance International, Maputo, Mozambique. "Emerging Bacterial Pathogens Unit, San Raffaele Scientific Institute, Milan, and ${ }^{f} \mathrm{WHO}$ Collaborating Centre for Tuberculosis and Lung Disease, S. Maugeri Foundation Care and Research Institute, Tradate, Italy. ${ }^{* * W o r l d}$ Health Organization, Geneva, Switzerland.

Correspondence: M. Zignol, STOP TB Dept, World Health Organization, 20 Avenue Appia 1211, Geneva 27, Switzerland. E-mail: zignolm@who.int

Statement of Interest: None declared.

\section{REFERENCES}

1 World Health Organization. Global Tuberculosis Control 2010 (WHO/HTM/TB/2010.7). Geneva, WHO, 2010.

2 MacArthur A, Gloyd S, Perdigao P, et al. Characteristics of drug resistance and HIV among tuberculosis patients in Mozambique. Int J Tuberc Lung Dis 2001; 5: 894-902.

3 World Health Organization. Multidrug and Extensively DrugResistant TB (M/XDR-TB) - 2010 Global Report on Surveillance and Response (WHO/HTM/TB/2010.3). Geneva, WHO, 2010.

4 Migliori GB, Besozzi G, Girardi E, et al. Clinical and operational value of the extensively drug-resistant tuberculosis definition. Eur Respir J 2007; 30: 623-626.

5 Sotgiu G, Ferrara G, Matteelli A, et al. Epidemiology and clinical management of XDR-TB: a systematic review by TBNET. Eur Respir J 2009; 33: 871-881.

6 World Health Organization. Guidelines for Surveillance of Drug Resistance in Tuberculosis. 2nd Edn (WHO/CDS/2003.320). Geneva, WHO, 2003.

7 Chonde TM, Basra D, Mfinanga SGM, et al. National antituberculosis drug resistance study in Tanzania. Int J Tuberc Lung Dis 2010; 14: 967-972.

8 Wright A, Zignol M, Van Deun A, et al. Epidemiology of antituberculosis drug resistance 2002-07: an updated analysis of the Global Project on Anti-Tuberculosis Drug Resistance Surveillance. Lancet 2009; 373: 1861-1873.

9 Cohen T, Murray M, Wallengren K, et al. The prevalence and drug sensitivity of tuberculosis among patients dying in hospital in KwaZulu-Natal, South Africa: a postmortem study. PLoS Med 2010; 22: e1000296.

10 Gandhi NR, Moll A, Sturm AW, et al. Extensively drug-resistant tuberculosis as a cause of death in patients co-infected with tuberculosis and HIV in a rural area of South Africa. Lancet 2006; 368: 1575-1580. 